\title{
Variáveis Familiares Preditoras do Comportamento Anti-Social em Adolescentes Autores de Atos Infracionais
}

\author{
Janaína Thaís Barbosa Pacheco ${ }^{1}$ \\ Claudio Simon Hutz \\ Universidade Federal do Rio Grande do Sul
}

\begin{abstract}
RESUMO - O objetivo deste estudo foi investigar variáveis individuais e familiares preditoras do comportamento anti-social. Os participantes foram 148 adolescentes, autores de atos infracionais, e 163 adolescentes não-infratores. Os adolescentes responderam individualmente a uma entrevista estruturada, que investigou estratégias educativas parentais, variáveis familiares e aspectos relacionados ao desenvolvimento do comportamento infrator. Uma análise de conteúdo das respostas permitiu a delimitação das principais práticas educativas relatadas pelos jovens. Uma análise de regressão indicou que o comportamento anti-social de familiares, o número de irmãos, o uso de drogas pelo adolescente, os conflitos na família e as práticas educativas parentais explicaram 53\% da variância do comportamento infrator. Os resultados apontaram a importância da família no desenvolvimento da conduta infratora.
\end{abstract}

Palavras-chave: adolescente; comportamento infrator; práticas educativas.

\section{Family Variables that Predict Antisocial Behavior in Adolescents who Committed Criminal Transgressions}

\begin{abstract}
The aim of the present study was to investigate individual and family variables that might predict delinquent behavior. The participants were 148 adolescents, institutionalized for committing criminal transgressions, and 163 adolescents who did not commit criminal transgressions. The participants were interviewed about parental practices, family variables, and other factors related to the development of the delinquent behavior. A content analysis of the answers allowed the delimitation of the main educational practices identified by the adolescents. Regression analysis showed that antisocial behavior of family members, number of siblings, drug use, conflicts within the family, and parental educational practices explained 53\% of the variance of delinquent behavior. These results pointed to the importance of the family in the development of the delinquent behavior.
\end{abstract}

Keywords: adolescents; delinquent behavior, educational practices.

O comportamento anti-social pode ser definido como um padrão de respostas cuja consequência é maximizar gratificações imediatas e evitar, ou neutralizar, as exigências do ambiente social; sua ocorrência estaria diretamente relacionada à ação de uma outra pessoa (Deater-Deckard \& Plomin, 1999; Patterson, Reid \& Dishion, 1992; Pettit, Laird, Dodge, Bates \& Criss, 2001). Patterson e colaboradores propõem que esse padrão é aprendido na infância e sugerem que tanto o comportamento pró-social quanto o comportamento anti-social de uma criança são diretamente produzidos por interações sociais, particularmente com membros da família, e vão se alterando a partir das exigências ambientais e do desenvolvimento do indivíduo (Capaldi \& Patterson, 1991; DeBaryshe, Patterson \& Capaldi, 1993; Patterson \& cols., 1992).

Outro aspecto importante na definição de comportamento anti-social é sua função na relação do indivíduo com o ambiente social. Os comportamentos anti-sociais de uma criança, por exemplo, moldam e manipulam o ambiente e, devido

1 Endereço para correspondência: Rua Miguel Tostes, 566/403. Porto Alegre, RS. CEP 90430-060. Tel: (51) 99632213. E-mail: janainapacheco@uol.com.br. a sua efetividade, podem tornar-se a principal forma desses indivíduos interagirem e lidarem com as outras pessoas (Patterson \& cols., 1992). Além disso, os pais, inadvertidamente, parecem reforçar tais condutas por meio de práticas parentais ineficazes (Capaldi, Chamberlain \& Patterson, 1997; Patterson, DeGarmo \& Knutson, 2000).

Patterson (1998) afirma que, no decorrer das interações, a criança e os outros membros da família vão gradualmente escalando na intensidade e na amplitude dos comportamentos coercitivos. Dessa forma, os comportamentos anti-sociais que ocorrem na infância são protótipos de comportamentos delinquentes $^{2}$ que poderão acontecer mais tarde. A delinquência,

2 Este estudo utilizou como critério de definição do comportamento delinquente o fato do jovem estar cumprindo medida sócio-educativa privativa de liberdade, prevista na legislação brasileira. No Brasil, desde a promulgação do Estatuto da Criança e do Adolescente - ECA (Brasil, 1991), utiliza-se o termo atos infracionais para designar os delitos cometidos por adolescentes. Optou-se por manter os termos anti-social e delinquente referindo-se ao comportamento desses jovens, porque a literatura consultada mantém a utilização de tais expressões. 
então, é um elemento de um amplo padrão anti-social que se inicia na infância e, normalmente, persiste na adolescência e adultez (Veirmeiren, 2003).

Para Scaramella, Conger, Spoth e Simons (2002), a distinção entre comportamento anti-social e delinquente é que o primeiro pode ou não incluir a violação de leis, enquanto o segundo refere-se, especificamente, a essas violações, como roubo, vandalismo ou violência contra outras pessoas. Nesse sentido, o comportamento delinquente é comumente definido por critérios jurídicos, uma vez que decorre da transgressão de normas codificadas (Gomide, 2004), e avaliado a partir de registros oficiais, em geral, ocorrências policiais, e de auto-relatos (Capaldi \& Patterson, 1991).

A estabilidade do comportamento anti-social tem sido investigada por uma série de estudos longitudinais que buscam compreender as variáveis que contribuem para a manutenção e a escalada desse padrão comportamental (Dishion, Patterson, Stoolmiller \& Skinner, 1991; Vuchinich, Bank \& Patterson, 1992). Ao tentar explicar as modificações que acontecem ao longo do tempo, Patterson e cols. (1992) propuseram o Modelo da Coerção ${ }^{3}$, que relaciona diversos fatores que contribuem para a evolução do comportamento anti-social e suas características em cada fase do desenvolvimento.

Patterson e cols. (1992) assinalam que, embora os estágios do Modelo da Coerção indiquem uma progressão, isso não significa que toda a criança anti-social irá escalar e manter esse padrão comportamental durante seu desenvolvimento. No entanto, a partir desse modelo teórico e de alguns estudos é possível identificar variáveis que favoreceriam a continuidade do comportamento anti-social e que podem ser consideradas como preditoras desse padrão comportamental na adolescência (Vuchinich \& cols., 1992). Entre elas, além de variáveis individuais como auto-estima e depressão, destacam-se: (a) a ocorrência de comportamento anti-social em pelo menos um dos pais (Patterson \& cols., 2000); (b) a idade de início desse padrão (Frick, Christian \& Wooton, 1999; Sourander, Helstelä, Helenius \& Piha, 2000) e a sua ocorrência em mais de um ambiente (Silva \& Rosseti-Ferreira, 2002); (c) as práticas educativas parentais (DeBaryshe \& cols., 1993; Pettit, Bates \& Dodge, 1997).

As estratégias utilizadas pelos pais, com o objetivo de promover a socialização de seus filhos, constituem o que a literatura refere como práticas educativas, disciplinares ou de cuidado (Hoffman, 1975, 1979, 1994). Os pais normalmente utilizam uma combinação de vários métodos e irão variá-los de acordo com a situação. Além disso, os métodos também irão entrelaçar-se com um conjunto de atitudes e sentimentos parentais, não necessariamente todos consistentes entre si (Grusec \& Lytton, 1988).

As práticas parentais têm sido classificadas de diversas formas pelos autores da área (Capaldi \& cols., 1997; Hoffman, 1975, 1979; Patterson \& cols., 1992). Hoffman $(1975,1979,1994)$ divide as práticas educativas parentais em técnicas coercitivas e indutivas. Entre as técnicas coercitivas podem ser citados o uso de punição verbal ou física,

3 Ver descrição do Modelo da Coerção em Pacheco, J., Alvarenga, P., Reppold, C. Piccinini, C. e Hutz, C. (2005). a privação de privilégios e a ameaça de castigo. Por outro lado, estratégias como o uso de explicação, o comando verbal não coercitivo e a alteração da situação ambiental são classificados como práticas indutivas (Alvarenga \& Piccinini, 2001; Hoffman, 1975).

Os estudos realizados por Patterson e cols. (1992) indicam outras categorias de análise de práticas educativas parentais. Entre essas, destacam-se o monitoramento parental, a disciplina, a habilidade para resolução de problemas, o reforçamento e a supervisão parental.

O monitoramento refere-se ao controle do comportamento, no sentido de saber onde o filho está, com quem está e o que está fazendo. Essa estratégia tem-se mostrado importante, pois diminui o risco do engajamento de crianças e adolescentes em comportamentos anti-sociais (Patterson \& cols., 1992; Pettit \& cols., 2001). Prover um controle parental firme na infância reduz a necessidade de regras na adolescência, provendo uma distribuição mais sistemática do poder na família (Baurmrind, 1997).

Consistentemente, os estudos indicam que fatores estressores que ocorrem no ambiente familiar, como desemprego ou divórcio, podem mediar as estratégias parentais. Além disso, outras variáveis familiares como o número de irmãos, a monoparentalidade, a drogadição ou outras psicopatologias em membros da família também parecem influenciar as estratégias adotadas pelos pais, estando relacionadas à inconsistência, à negligência e ao emprego de punição (Patterson \& cols., 1992).

Uma questão que tem sido considerada parte da problemática envolvendo o comportamento anti-social na adolescência é o consumo de drogas. O abuso de substância é frequentemente encontrado em comorbidade em jovens com Transtorno de Conduta e tem sido considerado como parte da 'síndrome de problemas de comportamento na adolescência' (Vermeiren, 2003). Existem evidências de que o abuso ou a dependência de drogas, em adolescentes infratores, está relacionado à severidade do comportamento anti-social, ao emprego de maior violência, uso mais pesado de álcool, ocorrência de depressão e alta incidência de tentativa de suicídio (Vermeiren, 2003).

Considerando que o comportamento anti-social é determinado por fatores individuais, familiares e contextuais, esse trabalho realizou um recorte propondo-se a estudar esse padrão comportamental, enfatizando as variáveis familiares. Este artigo integra um estudo de doutorado que buscou comparar adolescentes infratores e não infratores quanto a variáveis familiares que podem estar relacionadas ao desenvolvimento do comportamento infrator. O objetivo específico deste artigo foi investigar as variáveis individuais e familiares preditoras do comportamento anti-social.

\section{Método}

\section{Participantes}

Participaram deste estudo 311 adolescentes divididos em dois grupos: Infrator e Não Infrator. O Grupo Infrator foi composto por 148 adolescentes do sexo masculino, au- 
tores de atos infracionais, que estavam cumprindo medida sócio-educativa privativa de liberdade, na Fundação de Atendimento Sócio-Educativo (FASE, antiga FEBEM-RS). A idade e a escolaridade médias desses jovens foi de 17,2 anos e $5^{\mathrm{a}}$ série, respectivamente. O Grupo Não Infrator foi constituído por 163 adolescentes, que não cometeram atos infracionais, estudantes do Ensino Fundamental e Médio em escolas públicas de Porto Alegre. A idade média desse grupo foi de 16,6 anos e a escolaridade foi $6^{\text {a }}$ série. Procurou-se emparelhar o Grupo Não Infrator com o Grupo Infrator considerando o nível socioeconômico, o local de moradia, a idade e a escolaridade. Houve uma pequena diferença entre os dois grupos com relação a idade e a escolaridade porque muitos adolescentes do Grupo Infrator estavam afastados da escola antes da internação.

Os atos infracionais pelos quais os adolescentes estavam cumprindo medida sócio-educativa na época da entrevista foram: contra o patrimônio $(78,8 \%)$, a pessoa $(18,2 \%)$ e a liberdade sexual (1,5\%), e relacionados a tóxico $(1,5 \%)$. A categorização dos delitos foi realizada considerando o proposto pelo Código Penal Brasileiro (Brasil, 2001).

\section{Instrumentos}

As variáveis individuais e familiares foram investigadas por meio de uma entrevista estruturada. A entrevista foi construída baseada nos instrumentos utilizados por Alvarenga (2000) e Assis (1999), tendo sido incluídos alguns itens utilizados em outros estudos. A entrevista foi composta por três situações estruturadas que buscavam investigar as práticas educativas utilizadas pelos pais diante de comportamentos específicos dos filhos. As situações envolviam, respectivamente, as seguintes condutas: desobediência (S1), mentira (S2) e envolvimento com o cometimento de delitos (S3). Nas três situações, e para todos os adolescentes, foi perguntado primeiro sobre a mãe e depois sobre o pai.

Além das práticas educativas parentais, a entrevista estruturada constituiu-se de questões que abordaram os seguintes temas: número de irmãos, uso de álcool, drogas ilícitas ou cometimento de delitos por algum membro da família e uso de drogas pelo adolescente.

\section{Procedimento}

Todas as entrevistas foram individuais. As entrevistas na FASE foram realizadas de acordo com a disponibilidade e o interesse do adolescente, respeitando as características organizacionais da instituição.

As escolas nas quais ocorreram as entrevistas do Grupo Não Infrator possuíam programas de Educação de Jovens e Adultos (EJA) ou ensino regular com classes para alunos repetentes e atendiam adolescentes de baixa renda. Participaram do estudo nove escolas públicas da cidade de Porto Alegre. Na escola, o pesquisador utilizava os registros disponíveis para identificar as turmas e os alunos que tinham as características desejadas, tomando como base o grupo de adolescentes infratores. A partir dessa seleção, os adolescentes eram convidados a participar da pesquisa.
Os adolescentes que concordavam em participar recebiam o Termo de Consentimento Livre e Esclarecido que deveria ser assinado pelos pais.

\section{Resultados}

As estratégias que os pais usam para lidar com o comportamento dos filhos foram investigadas a partir das situações estruturadas que compunham a entrevista. As respostas dadas, pelos adolescentes, às situações estruturadas, foram submetidas à Análise de Conteúdo (Bardin, 1977/1997). A seguir são descritas as práticas educativas delimitadas pela análise.

1. Não interferência (negligência) - os pais não realizavam nenhum tipo de intervenção diante do comportamento do filho.

2. Aconselhamento - os pais aconselhavam ou conversavam sobre o comportamento do jovem, seja no sentido de produzir uma modificação, de alertar para medidas de cuidado que o jovem deveria tomar ou de dar ordens direcionadas ao comportamento.

3. Reforçamento - refere-se ao emprego, por parte dos pais, de estratégias que reforçavam (material ou socialmente) o comportamento inadequado dos filhos. Não foi considerada para a categorização se os pais tinham a intenção de reforçar o comportamento.

4. Punição física - os pais utilizavam intervenções utilizando controle ou agressão física.

5. Castigo ou privação de privilégio material - os pais utilizavam estratégias com o objetivo de privar o jovem de coisas que ele gosta, a fim de castigá-lo, ou de fazer o jovem entrar em contato com as consequências aversivas de seu próprio comportamento.

6. Delegar responsabilidades para outros - os pais atribuíam a outras pessoas ou instituições a responsabilidade sobre o controle do comportamento do filho.

As práticas educativas parentais foram analisadas para pais e mães, separadamente, e em cada situação da entrevista (S1, S2 e S3). Para investigar o valor preditivo das variáveis familiares e individuais com relação ao comportamento infrator foi realizada uma Análise de Regressão Linear (método Enter). Foram incluídas na Análise de Regressão as variáveis que apresentaram diferenças significativas entre os grupos. Essas variáveis foram o uso de álcool e cometimento de delitos por algum membro da família; o número de irmãos; o uso de drogas pelo adolescente; e as seguintes práticas educativas: não interferência; aconselhamento, reforçamento, punição física, castigo ou privação de privilégio material e delegar responsabilidades para outros. As diferenças nessas variáveis entre os Grupos Infrator e Não Infrator são apresentadas na Tabela 1.

Tanto o uso de drogas, quanto o cometimento de delitos por algum familiar, foi relatado com maior frequência pelo Grupo Infrator. Os familiares mais referidos por usarem drogas foram o pai $(29,6 \%)$, os irmãos $(9,4 \%)$ e os tios $(10,7 \%)$. Já os mais mencionados por cometerem delitos foram irmãos $(14,7 \%)$, primos $(10,1 \%)$ e tios $(9,8 \%)$.

Foi encontrada diferença estatisticamente significativa entre os dois grupos quanto ao uso de drogas pelos partici- 
Tabela 1. Variáveis que apresentaram diferença de média ou frequência entre o Grupo Infrator e o Grupo Não Infrator.

\begin{tabular}{lccc}
\hline Variáveis & $\begin{array}{c}\text { Grupo } \\
\text { Infrator }\end{array}$ & $\begin{array}{c}\text { Grupo Não } \\
\text { Infrator }\end{array}$ & Resultado \\
\hline Uso de drogas por um familiar & $42,3 \%$ & $15,0 \%$ & $\mathrm{X}^{2}=27,8 ; \mathrm{gl}=1 ; \mathrm{p}<001$ \\
Cometimento de delito por um familiar & $54,5 \%$ & $21,6 \%$ & $\mathrm{X}^{2}=35,4 ; \mathrm{gl}=1 ; \mathrm{p}<001$ \\
Número de irmãos & 4,3 & 2,6 & $\mathrm{t}=7,0 ; \mathrm{gl}=300 ; \mathrm{p}<05$ \\
Uso de drogas pelo adolescente & $87,4 \%$ & $30,9 \%$ & $\mathrm{X}^{2}=95,8 ; \mathrm{gl}=1 ; \mathrm{p}<001$ \\
\hline Prática educativas parentais & & & \\
- Não interferência (S3-Pai) & $21,2 \%$ & $1,6 \%$ & $\mathrm{X}^{2}=21,9 ; \mathrm{gl}=1 ; \mathrm{p}<001$ \\
- Não interferência (S3-Mãe) & $4,9 \%$ & $0,7 \%$ & $\mathrm{X}^{2}=4,9 ; \mathrm{gl}=1 ; \mathrm{p}<05$ \\
- Aconselhamento (S3-Mãe) & $36,0 \%$ & $9,9 \%$ & $\mathrm{X}^{2}=27,5 ; \mathrm{gl}=1 ; \mathrm{p}<001$ \\
- Aconselhamento (S3-Pai) & $37,6 \%$ & $21,3 \%$ & $\mathrm{X}^{2}=6,8 ; \mathrm{gl}=1 ; \mathrm{p}<01$ \\
- Reforçamento (S2-Mãe) & $2,5 \%$ & $0,0 \%$ & $\mathrm{X}^{2}=3,8 ; \mathrm{gl}=1 ; \mathrm{p}<05$ \\
- Punição física (S1-Pai) & $27,7 \%$ & $7,8 \%$ & $\mathrm{X}^{2}=15,8 ; \mathrm{gl}=1 ; \mathrm{p}<001$ \\
- Punição física (S1-Mãe) & $9,5 \%$ & $1,9 \%$ & $\mathrm{X}^{2}=7,9 ; \mathrm{gl}=1 ; \mathrm{p}<01$ \\
- Punição física (S2-Pai) & $31,8 \%$ & $9,1 \%$ & $\mathrm{X}^{2}=18,3 ; \mathrm{gl}=1 ; \mathrm{p}<001$ \\
- Castigo ou privação de privilégio material (S3-Mãe) & $18,9 \%$ & $53,3 \%$ & $\mathrm{X}^{2}=34,1 ; \mathrm{gl}=1 ; \mathrm{p}<001$ \\
- Delegar responsabilidades para outros (S3-Mãe) & $4,1 \%$ & $13,2 \%$ & $\mathrm{X}^{2}=6,7 ; \mathrm{gl}=1 ; \mathrm{p}<01$ \\
\hline
\end{tabular}

Tabela 2. Resultados da análise de regressão.

\begin{tabular}{lcccc}
\hline Variáveis independentes & \multicolumn{3}{c}{ Comportamento Infrator } \\
\hline 1. Uso de drogas pelo adolescente & $\boldsymbol{R}$ & $\beta(\boldsymbol{B})$ & $\boldsymbol{R}^{2}$ & $\boldsymbol{S} \boldsymbol{C}$ \\
2. Número de irmãos & 0,55 & 0,36 & 0,31 & 0,35 \\
3. S3 - mãe - aconselhar/conversar & 0,62 & 0,21 & 0,38 & 0,33 \\
4. Envolvimento de um familiar com delito & 0,64 & 0,09 & 0,41 & 0,33 \\
5. S3 - mãe - castigo ou privação de privilégio & 0,66 & 0,12 & 0,43 & 0,32 \\
6. S2 - pai - punição física & 0,68 & 0,12 & 0,45 & 0,31 \\
7. S3 - mãe - delegar para outras pessoas & 0,69 & 0,11 & 0,47 & 0,31 \\
8. S3 - pai - não interferência & 0,69 & 0,10 & 0,48 & 0,31 \\
9. S1 - pai - punição física & 0,70 & 0,10 & 0,49 & 0,30 \\
10. S3 - mãe - não interferência & 0,71 & 0,10 & 0,50 & 0,30 \\
11. Uso de álcool por um familiar & 0,71 & 0,09 & 0,51 & 0,30 \\
12. S3 - pai - aconselhar/conversar & 0,78 & 0,09 & 0,52 & 0,30 \\
13. S2 - mãe - reforçamento & 0,72 & 0,09 & 0,52 & 0,30 \\
14. S1 - mãe - punição física & 0,73 & 0,08 & 0,53 & 0,30 \\
\hline
\end{tabular}


pantes. As drogas mais citadas pelos adolescentes do Grupo Infrator foram a maconha, a cocaína e o crack. Essas são drogas que interferem de forma importante na conduta e têm propriedades que podem motivar ações violentas (Minayo \& Deslandes, 1998).

O número de irmãos foi investigado, pois a literatura tem considerado que essa pode ser uma variável preditora do comportamento anti-social. Nossos resultados confirmam essa suposição, mostrando que o número médio de irmãos no Grupo Infrator foi substancialmente maior do que no Grupo Não Infrator.

As práticas educativas foram investigadas para o pai e a mãe separadamente e em três situações específicas (S1, S2 e S3). Os resultados indicaram que as práticas educativas "Não interferência", "Aconselhamento", "Reforçamento", e "Punição Física" foram mais frequentes no Grupo Infrator, enquanto que as práticas “Castigo ou privação de privilégio material" e "Delegar responsabilidades para outros" foram mais frequentes no Grupo Não Infrator.

Os resultados mostraram que 14 variáveis contribuíram para explicar 53\% da variância do comportamento infrator. Essas variáveis e os resultados da análise de regressão são apresentados na Tabela 2.

\section{Discussão}

Este estudo integrou uma pesquisa cujo objetivo geral foi investigar variáveis familiares relacionadas à construção do comportamento infrator e comparar adolescentes infratores com adolescentes não infratores quanto a essas variáveis. Entende-se que o comportamento infrator é determinado por diversos aspectos que incluem dimensões sociais, culturais, individuais e familiares (Gomide, 2004).

Com base, então, na multiplicidade de fatores relacionados ao comportamento anti-social e na limitação característica de qualquer estudo empírico, para esta pesquisa optou-se por delimitar a investigação às variáveis familiares, em especial ao comportamento anti-social na família e às práticas educativas. Essa escolha foi sustentada teoricamente pelo Modelo da Coerção, proposto por Patterson e cols. (1992), que enfatiza o papel do grupo familiar.

O objetivo deste estudo foi investigar as variáveis preditoras do comportamento infrator. As análises indicaram que o uso de drogas pelos adolescentes, o número de irmãos, o envolvimento de um familiar com delito, o uso de drogas por algum familiar e as práticas educativas parentais (aconselhamento; castigo ou privação de privilégio material; punição física; delegar responsabilidades para outras pessoas; não interferência; e reforçamento do comportamento inadequado) explicaram $53 \%$ da variância do comportamento infrator. Esses resultados são especialmente importantes, pois essas relações ainda não haviam sido detectadas em estudos brasileiros.

Em relação às práticas educativas relatadas pelos adolescentes, é importante salientar que as características específicas da amostra (adolescentes de classes populares) podem ter contribuído para que as categorias relativas à conduta parental apresentassem um perfil diferente do apontado pela literatura. O reforçamento, por exemplo, é considerada uma estratégia parental importante para a construção de repertórios comportamentais competentes (Sidman, 1989/1995). Essa premissa refere-se, obviamente, ao reforçamento de comportamentos adequados. Os resultados indicaram que as famílias inadvertidamente utilizam o reforço contingente a comportamentos de risco dos adolescentes, principalmente os infratores, como desobedecer ou passar dias fora de casa. Da mesma forma, a categoria "aconselhar/conversar", segundo a classificação de Hoffman $(1975,1979)$, é considerada uma prática indutiva e, portanto, desejável no repertório parental. São estratégias que solicitam a modificação do comportamento dos jovens sem utilizar ameaça ou coerção. No entanto, no grupo de adolescentes infratores investigados a prática parental de aconselhar não parece ter a função de evitar ou prevenir a conduta infratora, mas de auxiliar o jovem a se proteger de ser ferido ou preso durante o ato infracional. Além disso, em muitos casos os adolescentes demonstraram dar pouca importância às orientações dos pais, na medida em que não souberam referir o conteúdo do ‘conselho' parental.

As estratégias que incluem a privação de privilégio ou a colocação do adolescente em contato com as consequências de seu comportamento têm sido consideradas formas brandas de controle do comportamento, mas quando combinadas com monitoramento e reforçamento de conduta adequada, mostram-se efetivas no controle do comportamento (Baumrind, 1997; Capaldi \& cols., 1997; Patterson \& cols., 1992). Dentro dessa perspectiva, a estratégia educativa classificada como “castigo ou privação de privilégio material”, mesmo tendo um caráter coercitivo, é considerada como uma estratégia que pode diminuir a probabilidade de comportamentos inadequados ou prevenir a ocorrência desses, contribuindo para o desenvolvimento dos jovens. Essa afirmação pode ser corroborada pelos resultados aqui obtidos, na medida em que essa estratégia foi significativamente mais frequente nas mães do Grupo Não Infrator.

Não intervir, ou atribuir a outras pessoas ou a instituições a responsabilidade de interferir no comportamento dos adolescentes, também foram estratégias que se constituíram em preditoras do comportamento infrator. Essas práticas podem refletir a evitação dos pais de enfrentarem os filhos, a incapacidade ou a falta de alternativas para lidarem com o comportamento do jovem, ou a ausência de interesse em se envolverem com situações que podem ser geradoras de conflito. É importante observar que a estratégia de "não interferência" foi mais empregada pelos pais do Grupo Infrator, possivelmente pelas razões mencionadas anteriormente. No entanto, a estratégia de “delegar responsabilidade para outros", foi mais frequente nas mães do Grupo Não Infrator, na Situação 3, que trata sobre o cometimento de delito. Pode-se pensar que, ao responder essa questão, o adolescente tenha imaginado que essa seria uma situação tão grave para a sua família que a mãe precisaria pedir ajuda para resolvê-la.

A punição física empregada tanto pela mãe, quanto pelo pai, também é uma variável preditora do comportamento infrator. Uso da punição física pode estar relacionado ao consumo de drogas, pelo jovem ou pelos familiares, e à existência de conflitos na família. Além disso, os pais podem ter a crença de que o processo de disciplina e de socialização dos filhos passa necessariamente pelo uso de punições físicas 
e verbais, pois muitas vezes eles próprios foram educados dessa forma. No entanto, a punição física compromete significativamente o desenvolvimento, na medida em que gera emoções negativas, como raiva, ansiedade e medo, impede o estabelecimento de vínculos sadios (Sidman, 1989/1995) e, principalmente para esses jovens, oferece um modelo de resolução de problemas que poderá ser reproduzido em outras relações e ambientes.

Os resultados encontrados neste estudo sugerem as práticas educativas que contribuem favoravelmente para o desenvolvimento desses adolescentes e as práticas que podem prejudicar seu desenvolvimento. A estratégia "castigo ou privação de privilégio material” apresentou frequência maior no Grupo Não Infrator, podendo ser considerada uma prática que protege o desenvolvimento do jovem. Por outro lado, as práticas "não interferência”, "punição física” e "aconselhamento" tiveram maior frequência no Grupo Infrator, estando relacionadas a prejuízo desenvolvimental para o adolescente e a ocorrência de comportamento anti-social.

Além das práticas educativas, as variáveis "número de irmãos", "cometimento de delitos e uso de drogas por algum familiar" e "uso de drogas pelo adolescente" também foram consideradas preditoras do comportamento infrator e todas apresentaram média e frequência maior no Grupo Infrator. A relação entre número de irmãos e o comportamento antisocial inclui aspectos como atenção, cuidado e monitoramento dos pais e renda familiar. Dessa forma, entende-se que quanto maior a família, maior é o risco de práticas coercitivas ou negligentes e o afeto e a atenção são mais diluídos. Além disso, a renda per capita é reduzida, dificultando ainda mais o atendimento das necessidades básicas da família e do adolescente.

Alguns autores (e.g., Patterson \& cols, 1992) afirmam que a existência de comportamento anti-social na família é um importante preditor desse padrão na infância e na adolescência. O envolvimento de um familiar com o cometimento de delitos parece contribuir para a aprendizagem dessa conduta, por meio da modelação (Bandura, 1969/1979; Gomide, 2004) e do treino direto. Da mesma forma, o consumo de drogas por um membro da família contribui para essa aprendizagem, além de ser um fator importante para a ocorrência de conflitos e de violência familiar.

Finalmente, o consumo de drogas pelo adolescente tem sido considerado parte de um problema de atos infracionais e de violência envolvendo crianças e adolescentes (Minayo $\&$ Deslandes, 1998). O jovem envolvido com o uso de drogas pode cometer delitos por estar drogado, como forma de obter dinheiro para seu consumo, ou pelo envolvimento com o tráfico ilegal.

\section{Considerações Finais}

Este estudo investigou variáveis familiares preditoras do comportamento anti-social.A investigação dessas variáveis familiares em um grupo de adolescentes de nível socioeconômico baixo constituiu-se em um desafio metodológico e em um aspecto inovador deste trabalho. A maioria dos instrumentos disponíveis, bem como os construtos teóricos relacionados foram construídos para amostras de adolescen- tes com escolaridade adequada à idade e de nível socioeconômico médio.

Portanto, entende-se que a compreensão das especificidades das estratégias educativas e das relações que se estabelecem no âmbito familiar desses jovens será efetivamente possível na medida em que forem desenvolvidos instrumentos de avaliação adequados para esse grupo. Além disso, é preciso considerar que as proposições teóricas acerca da família precisam ser revistas, quando o objetivo é estudar famílias de classes populares. A análise da dinâmica dessas famílias à luz de conceitos construídos com base em características da classe média poderá contribuir para a perpetuação de uma visão preconceituosa de desestruturação.

As implicações dos resultados apontam para necessidade de implementação de políticas de assistência, de educação, de prevenção e de tratamento destinadas a essas famílias. Entende-se que os fatores que contribuíram para a predição do comportamento anti-social podem ser minimizados ou reduzidos por meio de intervenções eficientes.

Especialmente no que se refere às práticas educativas parentais, a possibilidade de intervenção pode ocorrer por meio da orientação ou treinamento de pais. O treinamento de pais tem sido empregado principalmente no tratamento de crianças e adolescentes que apresentam problemas de externalização, tais como birras, agressão e desobediência, sendo nessa área que apresenta maior apoio empírico (McMahon, 1996). Os modelos de orientação a pais estão disponíveis na literatura (Kernberg \& Chazan, 1991/1993; Marinho \& Silvares, 2001).

É preciso considerar que o comportamento anti-social ocorre dentro de um contexto social e econômico mais amplo. Além de intervenções pontuais, descritas anteriormente, políticas que privilegiem aspectos familiares e busquem reduzir a situação de exclusão em que muitas dessas famílias se encontram não podem deixar de ser mencionadas. Entende-se que os programas de intervenção que pretendam enfrentar efetivamente o problema do comportamento infrator devem atuar em todos os contextos no qual o jovem está inserido. As intervenções a nível individual não serão efetivas se não se buscar intervir nos cenários em que os jovens se desenvolvem, principalmente a família.

\section{Referências}

Alvarenga, P. (2000). Práticas educativas maternas e problemas de comportamento na infância. Dissertação de Mestrado, Universidade Federal do Rio Grande do Sul. Porto Alegre.

Alvarenga, P., \& Piccinini, C. (2001). Práticas educativas maternas e problemas de comportamento em pré-escolares. Psicologia: Reflexão e Crítica, 14, 449-460.

Assis, S. (1999). Traçando caminhos em uma sociedade violenta. Rio de Janeiro: Fiocruz.

Bandura, A. (1979). Modificação do comportamento. (E. Nick \& L. Peota, Trad.) Rio de Janeiro: Interamericana (Trabalho original publicado em 1969).

Bardin, L. (1997). Análise de conteúdo. (L.A. Reto \& A. Pinheiro, Trad.). Lisboa: Edições 70 (Trabalho original publicado em 1977). 
Baumrind, D. (1997). The discipline encounter: Contemporary issues. Aggression and Violent Behavior, 2, 321-335.

Brasil (1991). Estatuto da Criança e do Adolescente. São Paulo: Editora Revista dos Tribunais.

Brasil (2001). Código Penal Brasileiro (16 a ed.). São Paulo: Saraiva.

Capaldi, D., \& Patterson, G. (1991). Relation of parental transitions to boys' adjustments problems. I. Linear hypotesis. II. Mothers at risk for transitions and unskillied parenting. Developmental Psychology, 27, 489-504.

Capaldi, D., Chamberlain, P., \& Patterson, G. (1997). Ineffective discipline and conduct problems in males: Association, late adolescent outcomes and prevention. Agression and Violent Behavior, 2, 343-353.

Deater-Deckard, K., \& Plomin, R. (1999). An adoption study of the etiology of teacher and parent reports of externalizing behavior problems in middle childhood. Child Development, 70, 144-154.

DeBaryshe, B., Patterson, G., \& Capaldi, G. (1993) A performance model for academic achievement in early adolescent boys. Developmental Psychology, 29, 795-804.

Dishion, T., Patterson, G., Stoolmiller, M., \& Skinner, M. (1991). Family, school and behavioral antecedents to early adolescent involvement with antisocial peers. Developmental Psychology, 27, 172-180.

Frick, P., Christian, R., \& Wooton, J. (1999). Age trends in the association between parenting practices and conduct problems. Behavior Modification, 23, 106-128.

Gomide, P. (2004). Menor infrator: a caminho de um novo tempo. Curitiba: Juruá.

Grusec, J. E., \& Lytton, H. (1988). Social development: History, theory and research. New York: Springer-Verlang.

Hoffman, M. (1975). Moral internalization, parental power, and the nature of parent-child interaction. Developmental Psychology, 11, 228-239.

Hoffman, M. (1979). Development of moral thought, feeling and behavior. American Psychologist, 34, 959-966.

Hoffman, M. (1994). Discipline and internalization. Developmental Psychology, 30, 26-28.

Kernberg, P., \& Chazan S. (1993). Crianças com transtorno de comportamento: manual de psicoterapia. (D. Batista, Trad.). Porto Alegre: Artes Médicas (Trabalho original publicado em 1991).

Marinho, M. L., \& Silvares, E. F. (2001). Modelos de orientação a pais de crianças com queixas diversificadas. Em R. C. Wielenska (Org.), Sobre comportamento e cognição, Vol. 6-Questionando e ampliando a teoria e as intervenções clínicas e em outros contextos (pp. 165-178). Santo André: Arbytes.

McMahon, R. (1996). Treinamento de pais. Em V. Caballo (Org.), Manual de técnicas de terapia e modificação do comportamento (pp. 399-422) (M. D. Claudino, Trad). São Paulo: Santos (Trabalho original publicado em 1996).
Minayo, M. C., \& Deslandes, S. (1998). A complexidade das relações entre drogas e álcool e violência. Cadernos de Saúde Pública, 14, 35-42.

Pacheco, J., Alvarenga, P., Reppold, C., Piccinini, C., \& Hutz, C. (2005). Estabilidade do comportamento anti-social na transição da infância para a adolescência: uma perspectiva desenvolvimentista. Psicologia: Reflexão e Crítica, 18, 55-61.

Patterson, G. (1998). Coercion as a basis for early age of onset for arrest. Em J. McCord (Ed.), Coercion and punishment in longterm perspectives (pp. 81-105). Cambridge: University Press.

Patterson, G. R., DeGarmo, D. S., \& Knutson, N. (2000). Hyperactive and antisocial behaviors: Comorbid or two points in the same process? Development and Psychopathology, 12, 91-106.

Patterson, G. R., Reid, J., \& Dishion, T. (1992). Antisocial boys. Eugene: Castalia.

Pettit, G. S., Bates, J. E., \& Dodge, K. A. (1997). Supportive parenting, ecological context, and children's adjustment: A sevenyear longitudinal study. Child Development, 68, 908- 923.

Pettit, G. S., Laird, R., Dodge, K., Bates, J. E., \& Criss, M. (2001). Antecedents and behavior-problem outcomes of parental monitoring and psychological control in early adolescence. Child Development, 72, 583-598.

Scaramella, L., Conger, R., Spoth, R., \& Simons, R. (2002). Evaluation of a social contextual model of delinquency: A crossstudy replication. Child Development, 73, 175-195.

Sidman, M. (1995). Coerção e suas implicações. (M.A. Andery \& T.M. Sério, Trad.) São Paulo: Editorial Psy II (Trabalho original publicado em 1989).

Silva, A. P. \& Rossetti-Ferreira, C. (2002). Continuidade / descontinuidade no envolvimento com o crime: uma discussão crítica da literatura na psicologia do desenvolvimento. Psicologia: Reflexão e Crítica, 15, 573-585.

Sourander, A., Helstelä, L., Helenius, H., \& Piha, J. (2000). Persistence of bullying from childhood to adolescence - A longitudinal 8-years follow-up study. Child Abuse \& Neglect, 24, 873-881.

Veirmeiren, R. (2003). Psycopathology and delinquency in adolescents: A descriptive and developmental perspective. Clinical Psychology Review, 23, 277-318.

Vuchinich, S., Bank, L., \& Patterson, G. (1992). Parenting, peers and the stability of antisocial behavior in preadolescent boys. Developmental Psychology, 28, 510-521. 